\title{
Ultralow-Threshold and Color-Tunable Continuous-Wave Lasing at Room-Temperature from In Situ Fabricated Perovskite Quantum Dots
}

Lei Wang, ${ }^{\dagger}$ Linghai Meng, ${ }^{\dagger}$ Lan Chen,${ }^{\ddagger}$ Sheng Huang, ${ }^{\dagger}$ Xiangang Wu, ${ }^{\dagger}$ Guang Dai, ${ }^{\S}$ Luogen

Deng, ${ }^{\S}$ Junbo Han, ${ }^{\|}$Bingsuo Zou, ${ }^{\S}$ Chunfeng Zhang, ${ }^{\ddagger}$ Haizheng Zhong ${ }^{*},+$

${ }^{\dagger}$ Beijing Key Laboratory of Nanophotonics and Ultrafine Optoelectronic Systems, School of Materials Science \& Engineering, Beijing Institute of Technology, 5 Zhongguancun South Street, Haidian District, Beijing 100081, China

${ }^{\ddagger}$ National Laboratory of Solid State Microstructures, School of Physics \& Collaborative Innovation Center of Advanced Microstructures, Nanjing University, Nanjing 210093, China

${ }^{\S}$ School of Physics, Beijing Institute of Technology, 5 Zhongguancun South Street, Haidian District, Beijing 100081, China

"Wuhan National High Magnetic Field Center and Department of Physics, Huazhong University of Science and Technology, Wuhan 430074, China 


\section{Experimental details}

\section{Materials:}

All reagents were of analytical grade and used as received without further purification. PAN (Mn = 150 000, Kynar Flex Plastic Materials Supplied by Arkema), $\mathrm{CH}_{3} \mathrm{NH}_{2}$ (methylamine, 33 wt $\%$ in absolute ethanol, Aladdin), $\mathrm{PbBr}_{2}$ (lead(II) bromide 99\%, Aladdin), $\mathrm{PbCl}_{2}$ (lead(II) chloride, 99\%, Alfa Aesar), $\mathrm{PbI}_{2}$ (lead(II) iodide 98.5\%, Alfa Aesar), DMF (analytical grade, Beijing Chemical Reagent Co., Ltd., China), HBr (hydrobromic acid, 48 wt\% in water, Aladdin), $\mathrm{HCl}$ (hydrochloric acid, $37 \mathrm{wt} \%$ in water, Aladdin), and $\mathrm{HI}$ (hydriodic acid, $57 \mathrm{wt} \%$ in water, Alfa Aesar).

\section{Synthesis of $\mathrm{CH}_{3} \mathrm{NH}_{3} \mathrm{X}(\mathrm{X}=\mathrm{Cl}, \mathrm{Br}$, or I):}

$\mathrm{CH}_{3} \mathrm{NH}_{3} \mathrm{X}(\mathrm{X}=\mathrm{Cl}, \mathrm{Br}$, or I) was synthesized by reaction of the methylamine with the corresponding acid. Firstly, methylamine in absolute ethanol was stirred and cooled to $0{ }^{\circ} \mathrm{C}$ with the addition of acid. The reaction solution was stirred for $2 \mathrm{~h}$. Then the solvent was evaporated in a rotary evaporation with a pressure of $-0.1 \mathrm{Mpa}$ at $45 \mathrm{C}$. At last, the precipitate was washed three times with diethylether and dried under vacuum $(60 \mathrm{C}, 5 \mathrm{~h})$ for future use.

\section{In situ fabrication of the PQD-embedded PAN films:}

The in situ fabrication process of the $\mathrm{MAPbBr}_{3}$-based PQD-embedded PAN films was similar to our previous work. ${ }^{1}$ Stage I: A precursor solution was prepared by adding $92 \mathrm{mg}$ MABr, 143 $\mathrm{mg} \mathrm{PbBr}_{2}$, and $1 \mathrm{~g} \mathrm{PAN}$ powder in $7 \mathrm{~mL} \mathrm{DMF}$. The precursor solution was stirred vigorously for $8 \mathrm{~h}$ until all visible opaque matter disappeared. The well-sealed precursor solution was spin-coated on the quartz glass substrate at $2000 \mathrm{rpm}$ for $30 \mathrm{sec}$ and dried in a vacuum oven for $30 \mathrm{~min}$ to remove the solution DMF. Under drying, the precursor films gradually changed into emissive composite films. The $\mathrm{MAPbBr} \times \mathrm{Cl}_{3-\mathrm{X}}$-based PQD-embedded PAN films (blue films) and the 
$\mathrm{MAPbI}_{3}-\mathrm{PQD}$-embedded PAN films (red films) were fabricated by varying the precursor solution constitution following a similar strategy.

\section{Time-resolved PL trace measurement:}

The time-resolved PL trace were recorded at the peak wavelength of the PL spectra by timecorrelated single-photon counting using an avalanche photodiode having a temporal resolution of $\sim 50$ ps. The wavelength of the pump light is $375 \mathrm{~nm}$ and the data are recorded with 16 ps per channel.

\section{Cavity design and fabrication:}

We simulated the first-order, second-order and fifth-order gratings on $\mathrm{SiO}_{2}$ substrate by applying COMSOL Multiphysics software. To form first-order laser mode, the grating period, the grating height and the thickness of the gain layer are fixed at $176 \mathrm{~nm}, 100 \mathrm{~nm}$ and $500 \mathrm{~nm}$ respectively (Figure S6). Similarly, the second-order grating requires a grating period of $341 \mathrm{~nm}$, grating height of $300 \mathrm{~nm}$ and gain layer thickness of $700 \mathrm{~nm}$ respectively (Figure S7). Because the viscosity of the perovskite/polymer precursor solution is quite high, it is not possible to obtain very flat thin PQD-PAN films less than $1.5 \mu \mathrm{m}$. Therefore, we used fifth order grating to achieve CW lasing. For the fifth-order DFB gratings, the period of the gratings for the green, blue and red lasers are $866 \mathrm{~nm}, 770 \mathrm{~nm}$ and $1020 \mathrm{~nm}$ respectively (Figure S8). The cavities were fabricated using electron beam lithography (EBL). We first deposited $50 \mathrm{~nm}$ aluminum layer on $\mathrm{SiO}_{2}$ by magnetron sputtering method. After EBL resist patterning, the aluminum layer and $\mathrm{SiO}_{2}$ were etched to the depth met the requirements. The residual aluminum and resist was then removed using diluted $\mathrm{HCl}$ (10\%) chemistries. The perovskite/polymer solution was spin-coated on the cavities and dried for 30 min. After removing the solution DMF, the PQDs embedded PAN films were deposited on the cavities. 


\section{Calculations:}

The calculation of the charge density at CBM and VBM of the system was based on the density functional theory (DFT), which was performed by the PWmat code, a DFT Plane Wave materials simulation code run on GPU clusters. The pseudo potential adopted the NCPP-SG15-PBE. ${ }^{2,3}$ In our models, the value of the vacuum regions was $8 \AA$ and the number of layer $\mathrm{N}$ equaled to 5 . $\mathrm{K}$ point grids of $2 \times 2 \times 2$ were chosen for all systems. To simplify the model, we substituted the ligand acetonitrile, methylamine and vinylidene fluoride group for the PAN, n-octylamine and PVDF respectively. The $\mathrm{MAPbBr}_{3} / \mathrm{Ligand}$ interfaces were modeled by placing ligand on top of the $\mathrm{MAPbBr}_{3}(001)$ surface. In order to decouple adjacent slabs, a thick vacuum layer of 15 Á was included in the direction perpendicular to the surface. 
Table S1. Thermal conductivities of some commercial polymers s.5 $^{4,5}$

\begin{tabular}{|c|c|}
\hline Material & $\begin{array}{l}\text { Thermal conductivity at room temperature } \\
\left(\mathrm{W} \mathrm{m}^{-1} \mathrm{~K}^{-1}\right)\end{array}$ \\
\hline Polymethylmethacrylate (PMMA) & 0.21 \\
\hline Polystyrene (PS) & 0.14 \\
\hline Polypropylene (PP) & 0.11 \\
\hline Poly(ethylene terephthalate) (PET) & 0.15 \\
\hline Poly(butylene terephthalate) (PBT) & 0.29 \\
\hline Polycarbonate (PC) & 0.20 \\
\hline Low density polyethylene (LDPE) & 0.30 \\
\hline High density polyethylene (HDPE) & 0.30 \\
\hline High density polyethylene (HDPE) & 0.44 \\
\hline Poly(acrylonitrile-butadiene-styrene) copolymer (ABS) & 0.33 \\
\hline Polyetheretherketone (PEEK) & 0.25 \\
\hline Polyphenylene sulfide (PPS) & 0.30 \\
\hline Polysulfone (PSU) & 0.22 \\
\hline Polyphenylsulfone (PPSU) & 0.35 \\
\hline Polyvinyl chloride (PVC) & 0.19 \\
\hline Polyvinylidene difluoride (PVDF) & 0.19 \\
\hline Polytetrafluoroethylene (PTFE) & 0.27 \\
\hline Poly(ethylene vinyl acetate) (EVA) & 0.34 \\
\hline Polyimide, Thermoplastic (PI) & 0.11 \\
\hline Poly(dimethylsiloxane) (PDMS) & 0.25 \\
\hline Polyamide-6,6 (PA66) & $0.24-0.33$ \\
\hline Polyamide-6 (PA 6) & $0.22-0.33$ \\
\hline Urethane base TPE (TPU) & 0.19 \\
\hline Styrene/polybutadiene copolymer (SB) & $0.17-0.18$ \\
\hline Styrene-acrylonitrile copolymer (SAN) & $0.15-0.17$ \\
\hline Styrene/polybutadiene copolymer (SB) & $0.17-0.18$ \\
\hline Polyisobutylene (PIB) & $0.12-0.20$ \\
\hline Ultrahigh molecular weight polyethylene (UHMWPE) & $0.41-0.51$ \\
\hline MAPbX $_{3}$ PQD-embedded PAN films (Our work) & 1.25 \\
\hline
\end{tabular}


Table S2. The CW lasers from solution-processed nanocrystals

\begin{tabular}{|c|c|c|c|c|c|}
\hline Materials & $\begin{array}{l}\text { Cavity } \\
\text { Type }\end{array}$ & Laser Color & Thresholds & Temperature & $\begin{array}{l}\text { Publication } \\
\text { Date (year) }\end{array}$ \\
\hline CdSe quantum wells ${ }^{6}$ & DBR & Green & $0.44 \mathrm{~kW} \mathrm{~cm}^{-2}$ & $\begin{array}{c}\text { Room } \\
\text { temperature }\end{array}$ & 2014 \\
\hline $\mathrm{MAPbl}_{3} \mathrm{film}^{7}$ & DFB & Red & $\approx 17 \mathrm{~kW} \mathrm{~cm}^{-2}$ & $102 \mathrm{~K}$ & 2017 \\
\hline CdSe/ZnS CQDs ${ }^{8}$ & WG & Red & $500 \mathrm{~kW} \mathrm{~cm}^{-2}$ & $\begin{array}{c}\text { Room } \\
\text { temperature }\end{array}$ & 2017 \\
\hline CsPbBr3 nanowires ${ }^{9}$ & FP & Green & $6 \mathrm{~kW} \mathrm{~cm}^{-2}$ & $77 \mathrm{~K}$ & 2017 \\
\hline CdSe/CdS CQDs ${ }^{10}$ & DFB & Red & $6.4-8.4 \mathrm{~kW} \mathrm{~cm}^{-2}$ & $\begin{array}{l}-20^{\circ} \mathrm{C} \\
(253 \mathrm{~K})\end{array}$ & 2017 \\
\hline $\begin{array}{c}\text { CdSe/CdS } \\
\text { nanoplatelets }{ }^{11}\end{array}$ & DFB & Red & $220 \mathrm{~W} \mathrm{~cm}^{-2}$ & $\begin{array}{c}\text { Room } \\
\text { temperature }\end{array}$ & 2017 \\
\hline $\begin{array}{c}\text { Charged } \\
\text { CdSe/CdSe } \mathrm{S}_{1-x} / \mathrm{CdS} \\
\mathrm{QDs}^{12}\end{array}$ & & Red & $\begin{array}{c}500 \mathrm{~kW} \mathrm{~cm}^{-2} \\
\text { (calculated value) }\end{array}$ & & 2017 \\
\hline $\begin{array}{c}\mathrm{MAPbl}_{3} \text { polycrystalline } \\
\text { film }^{13}\end{array}$ & DFB & Red & $13 \mathrm{~W} \mathrm{~cm}^{-2}$ & $\begin{array}{c}\text { Room } \\
\text { temperature }\end{array}$ & 2018 \\
\hline $\begin{array}{c}\text { MAPbX }_{3} \text { PQD- } \\
\text { embedded PAN films } \\
\text { (In situ fabrication) }\end{array}$ & DFB & $\begin{array}{l}\text { Color tunable } \\
\text { (green, blue, } \\
\text { red) }\end{array}$ & $\begin{array}{l}\text { Green: } 15 \mathrm{~W} \mathrm{~cm}^{-2} \\
\text { Red: } 24 \mathrm{~W} \mathrm{~cm}^{-2} \\
\text { Blue: } 58 \mathrm{~W} \mathrm{~cm}^{-2}\end{array}$ & $\begin{array}{c}\text { Room } \\
\text { temperature }\end{array}$ & Our work \\
\hline
\end{tabular}



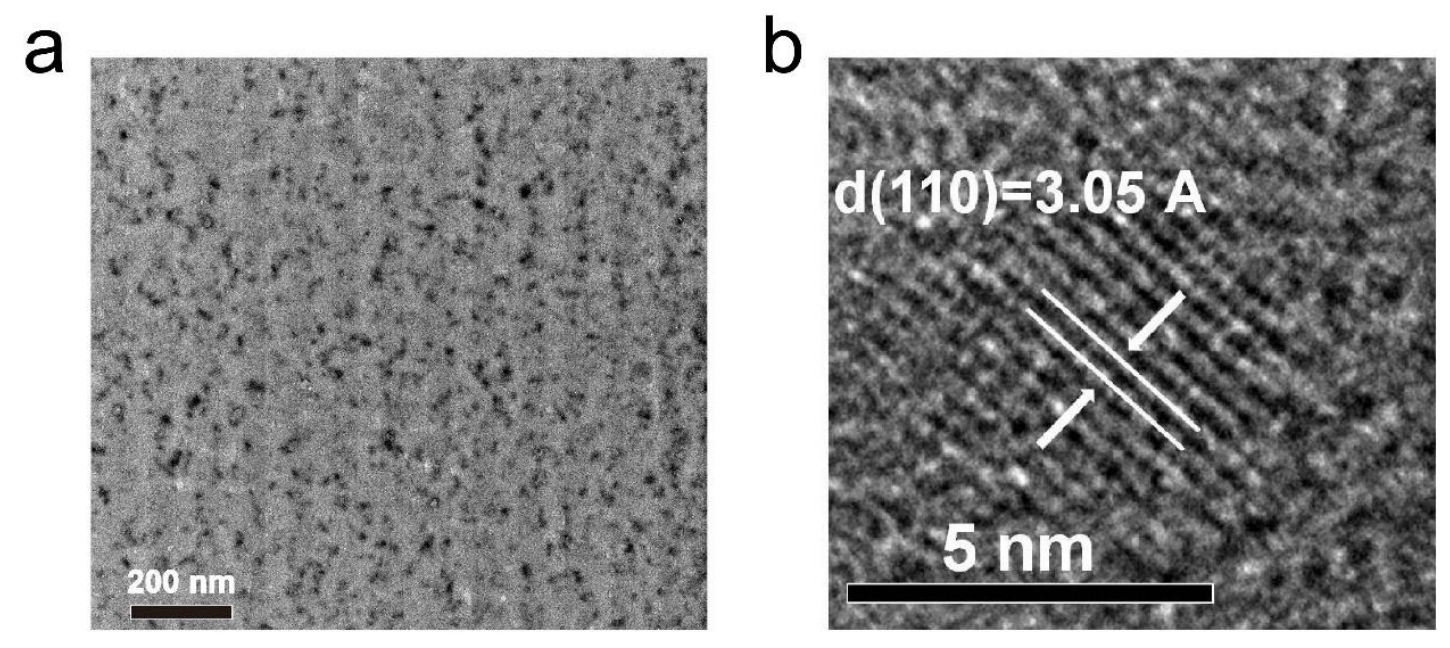

Figure S1. (a) TEM image of $\mathrm{MAPbBr}_{3} \mathrm{PQDs}$ in PAN matrix. (b) The high-resolution TEM image of a typical $\mathrm{MAPbBr}_{3} \mathrm{QD}$. The obvious lattice distances of $3.05 \AA$, corresponding to the lattice distance of (110) plane of $\mathrm{MAPbBr}_{3}$ QDs, can be clearly identified.

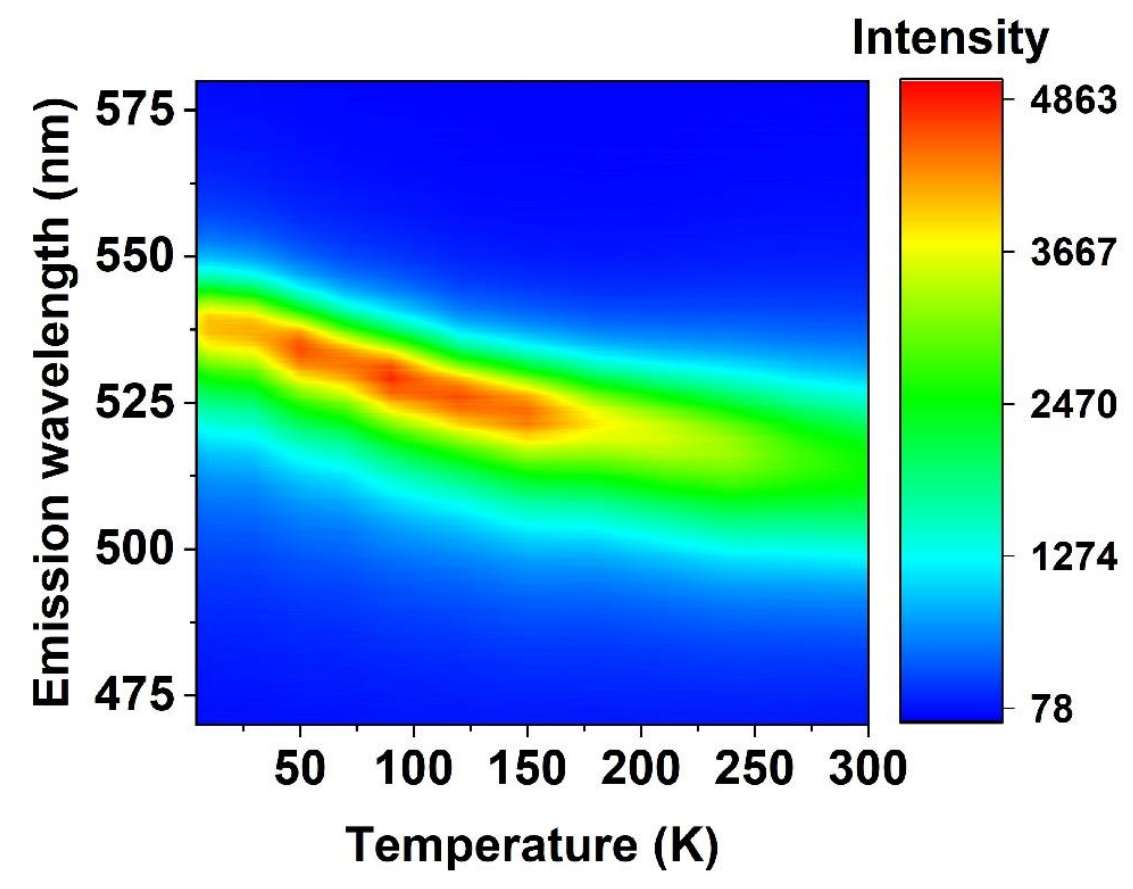

Figure S2. Pseudocolor map of temperature-dependent PL spectra of the $\mathrm{MAPbBr}_{3}$-based PQDembedded PAN film recorded from $4.2 \mathrm{~K}$ to $300 \mathrm{~K}$. 


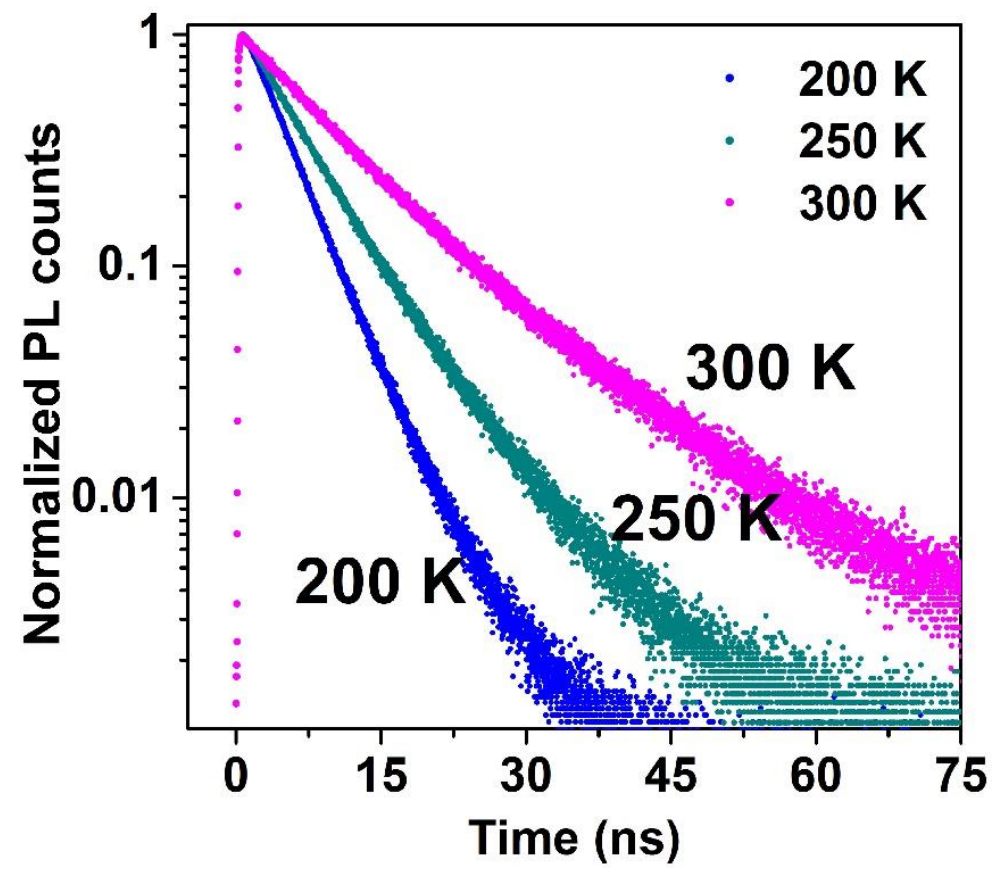

Figure S3. Time-resolved PL traces of the $\mathrm{MAPbBr}_{3}$-based PQD-embedded PAN film at temperatures ranging from $200 \mathrm{~K}$ to $300 \mathrm{~K}$. The decay curves can be fitted by monoexponential functions.

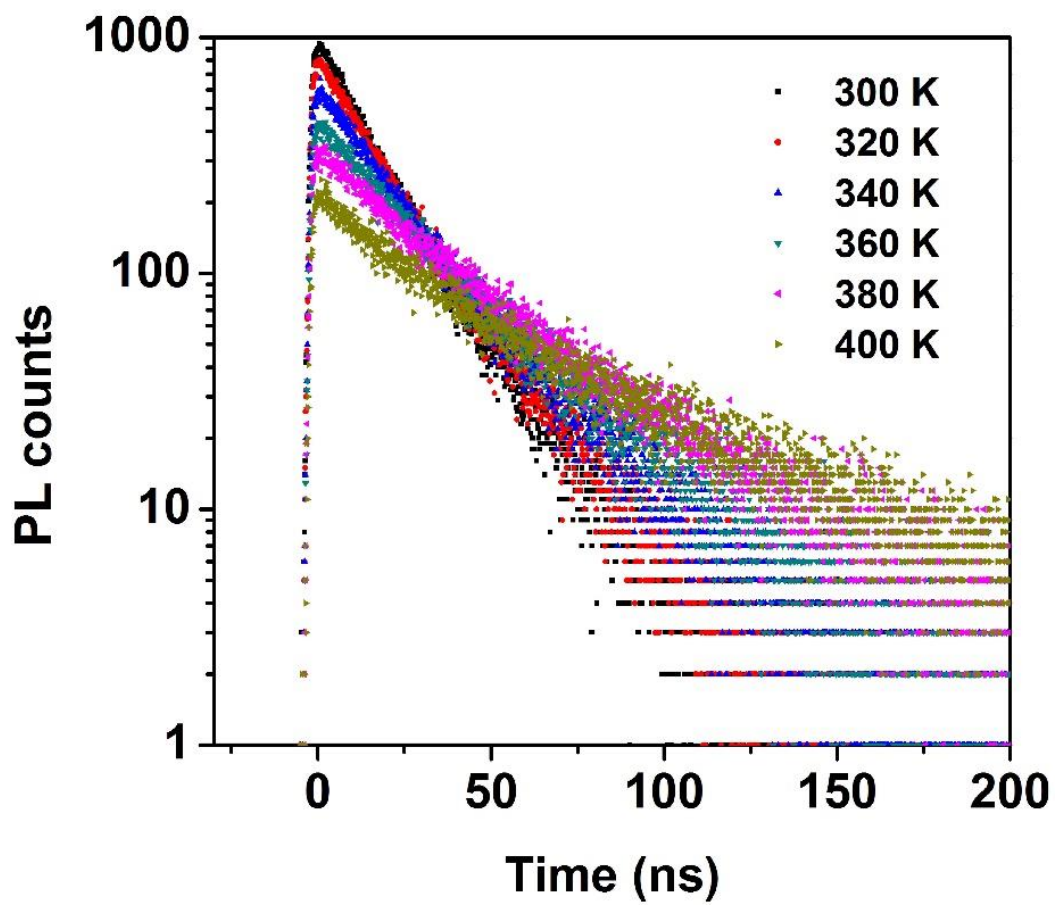

Figure S4. Time-resolved PL traces of the $\mathrm{MAPbBr}_{3}$-based $\mathrm{PQD}$-embedded $\mathrm{PAN}$ film at temperatures ranging from $300 \mathrm{~K}$ to $400 \mathrm{~K}$. the PL shows monoexponential decay at the temperature as high as $400 \mathrm{~K}$. 


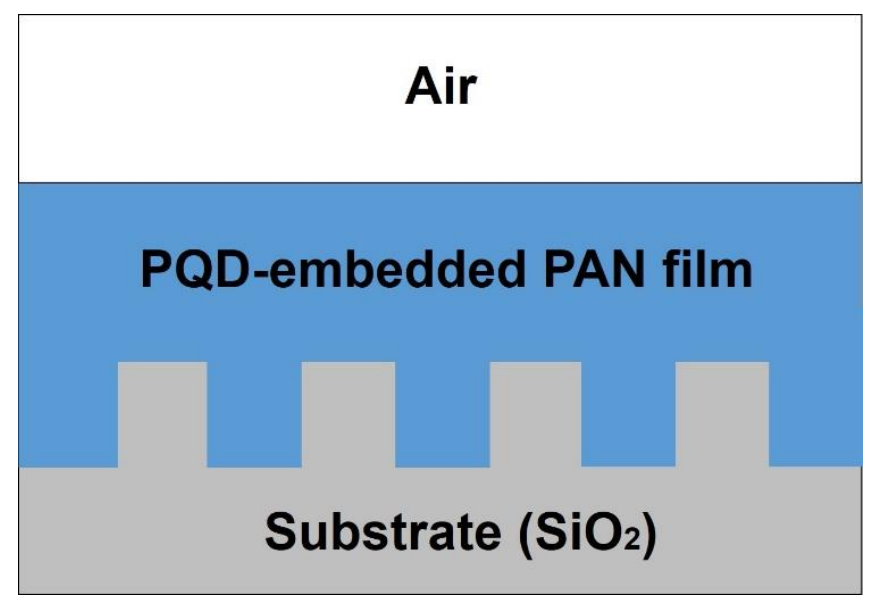

Figure S5. The structure of the DFB laser used in this paper.

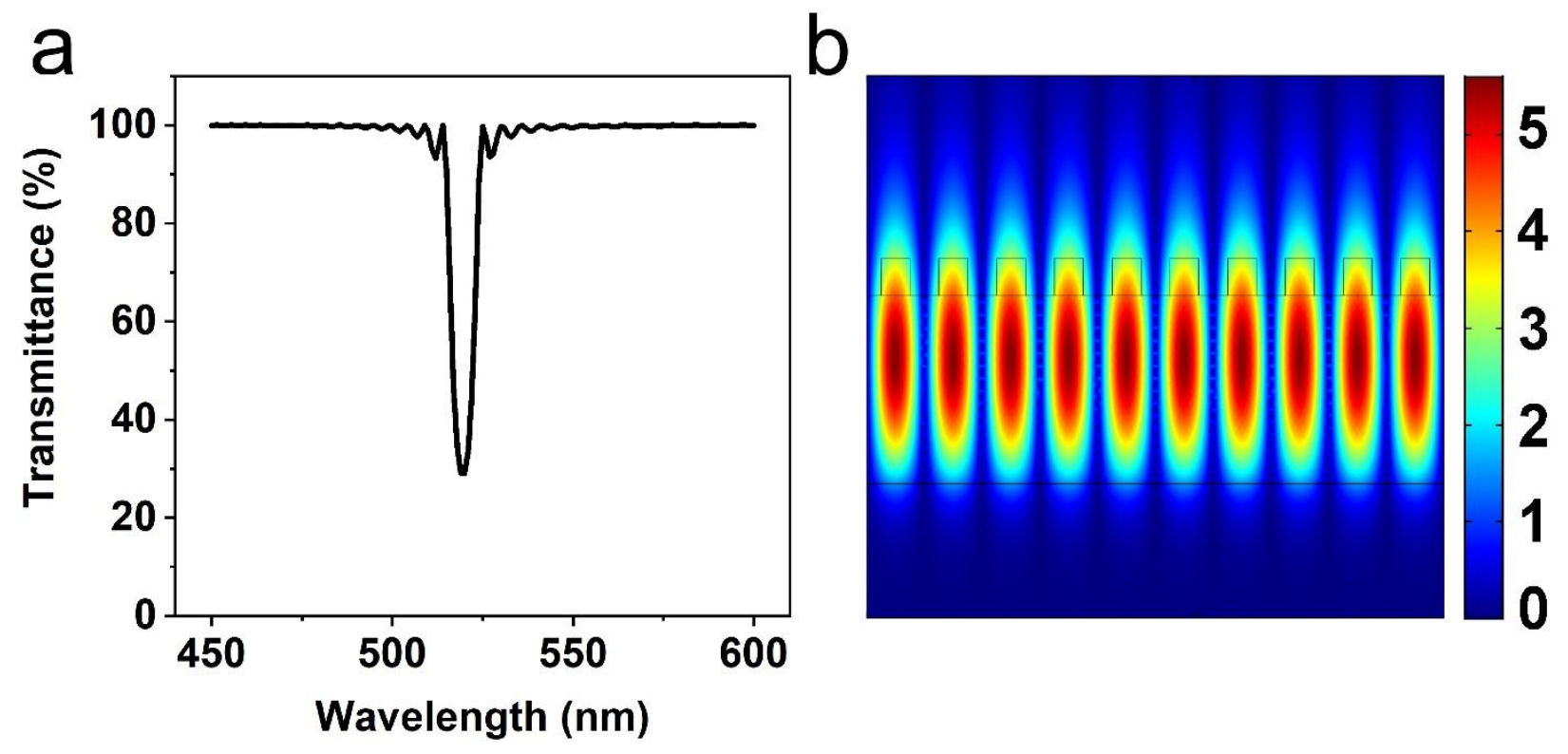

Figure S6. (a) The transmittance spectrum of the first-order grating and (b) the distribution of light field at peak wavelength. If the grating period, the grating height and the thickness of the gain layer thickness are fixed at $176 \mathrm{~nm}, 100 \mathrm{~nm}$ and $500 \mathrm{~nm}$ respectively, the optical field at $520 \mathrm{~nm}$ locates in the gain layer to form the first-order laser mode. 

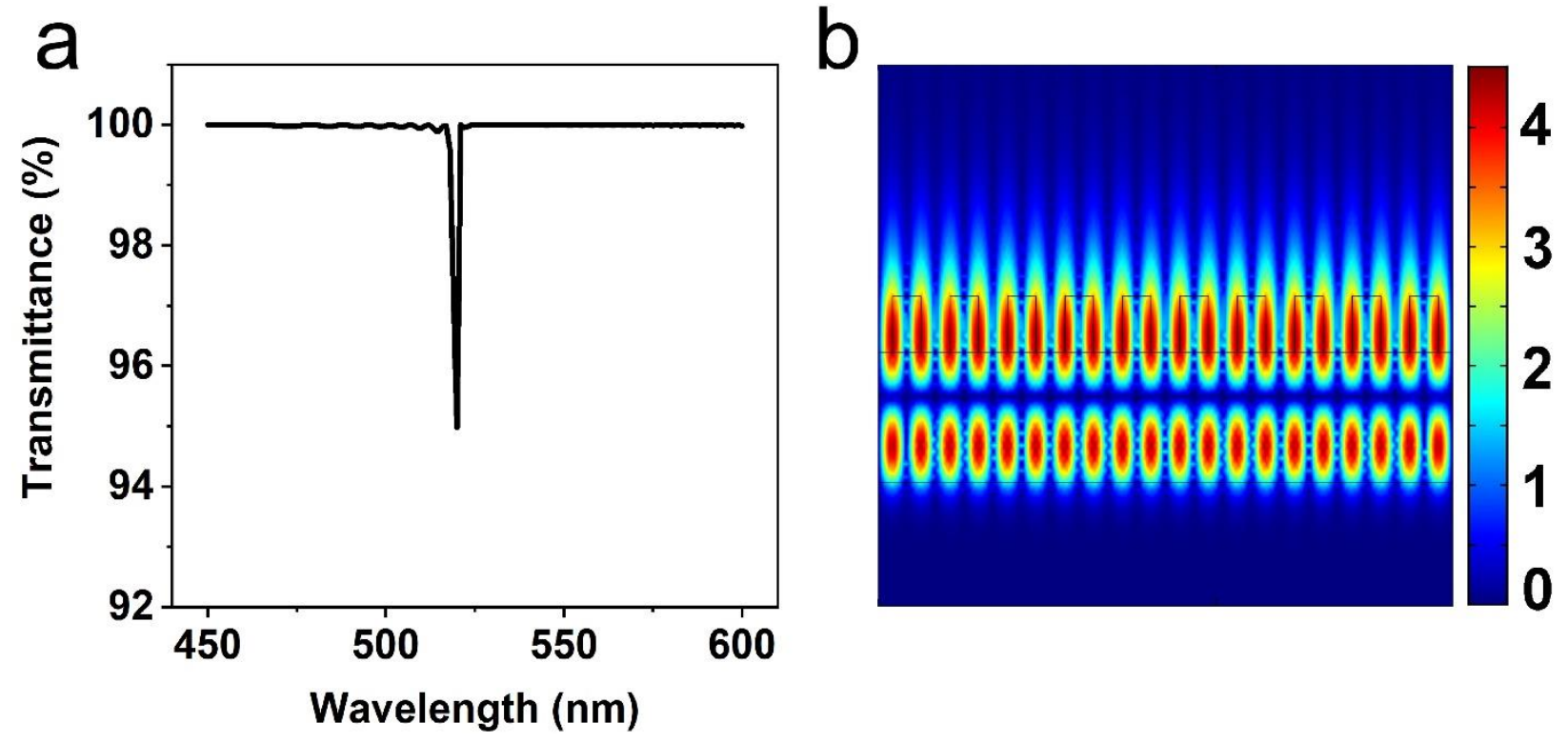

Figure S7. (a) The transmittance spectrum of the second-order grating and (b) the distribution of light field at peak wavelength. If the grating period, the grating height and the thickness of the gain layer thickness are fixed at $341 \mathrm{~nm}, 300 \mathrm{~nm}$ and $700 \mathrm{~nm}$ respectively, the optical field at $520 \mathrm{~nm}$ locates in the gain layer to form the second-order laser mode.

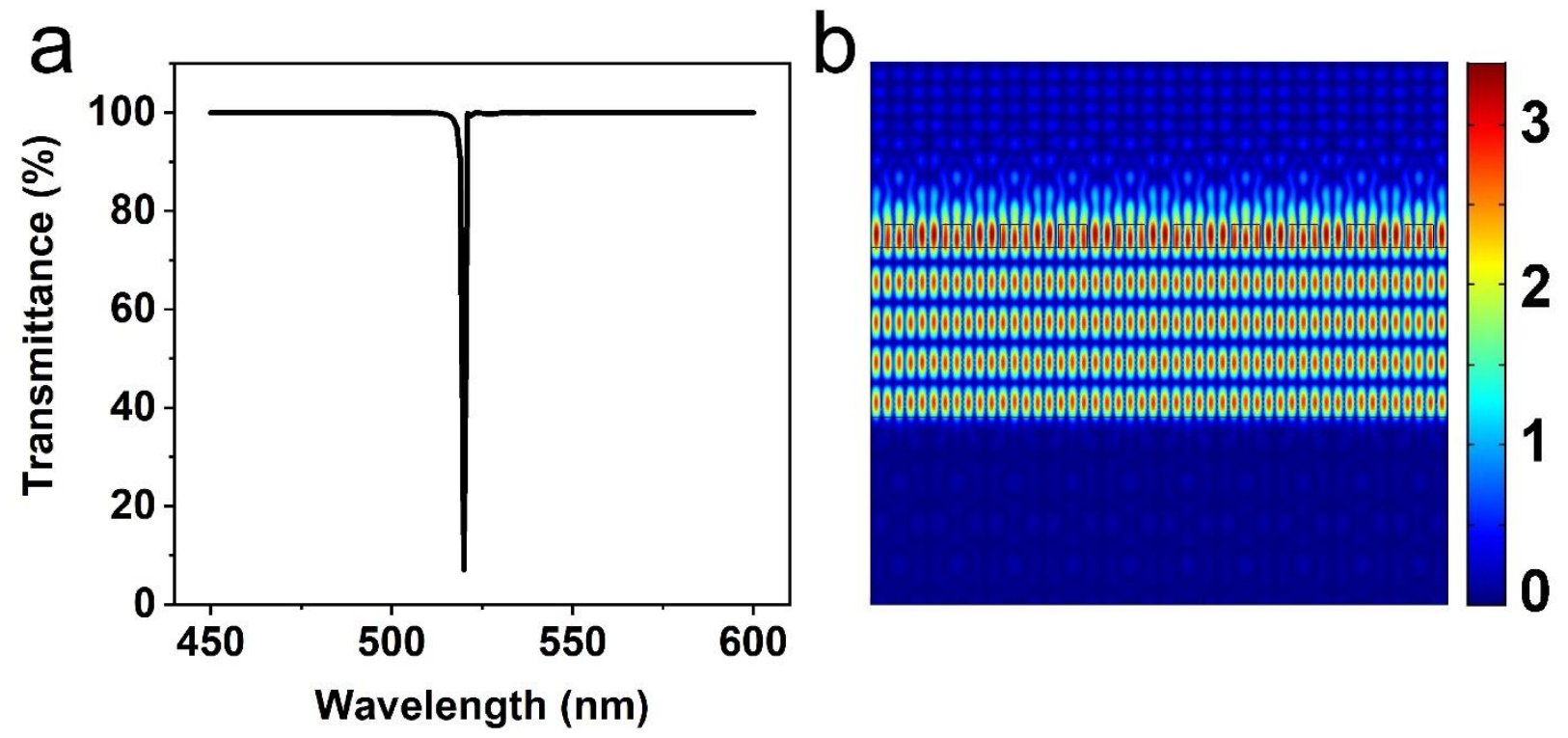

Figure S8. (a) The transmittance spectrum of the fifth-order grating and the (b) distribution of light field at peak wavelength. If the grating period, the grating height and the thickness of the gain layer thickness are fixed at $866 \mathrm{~nm}, 300 \mathrm{~nm}$ and $2200 \mathrm{~nm}$ respectively, the optical field at $520 \mathrm{~nm}$ locates in the gain layer to form the fifth-order laser mode. 


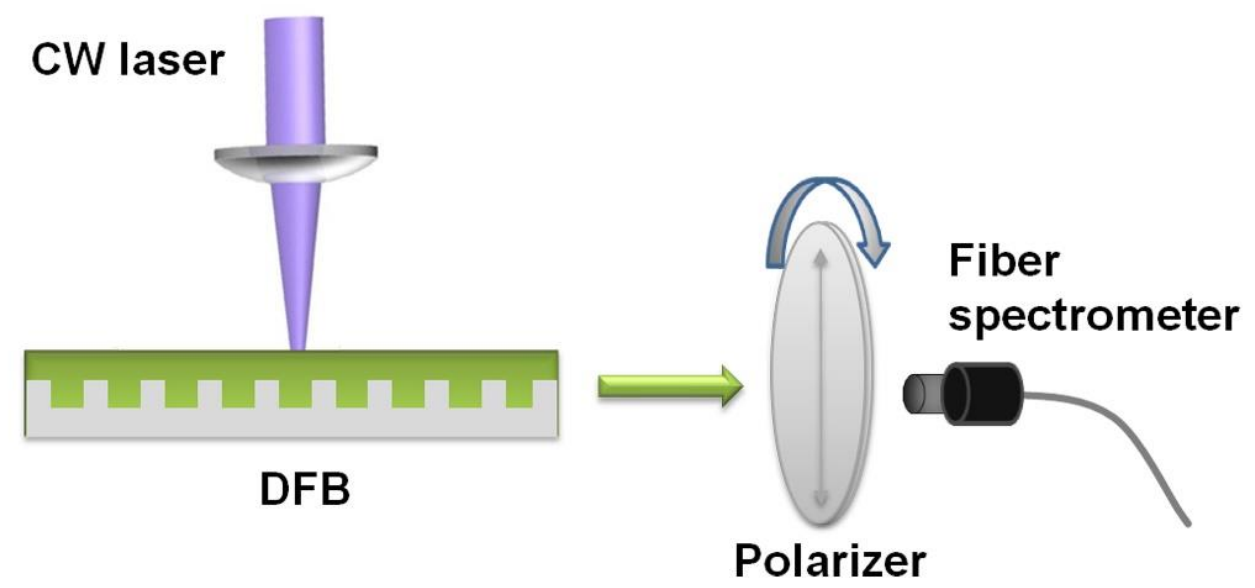

Figure S9. Schematic setup of the polarized lasing measurement.
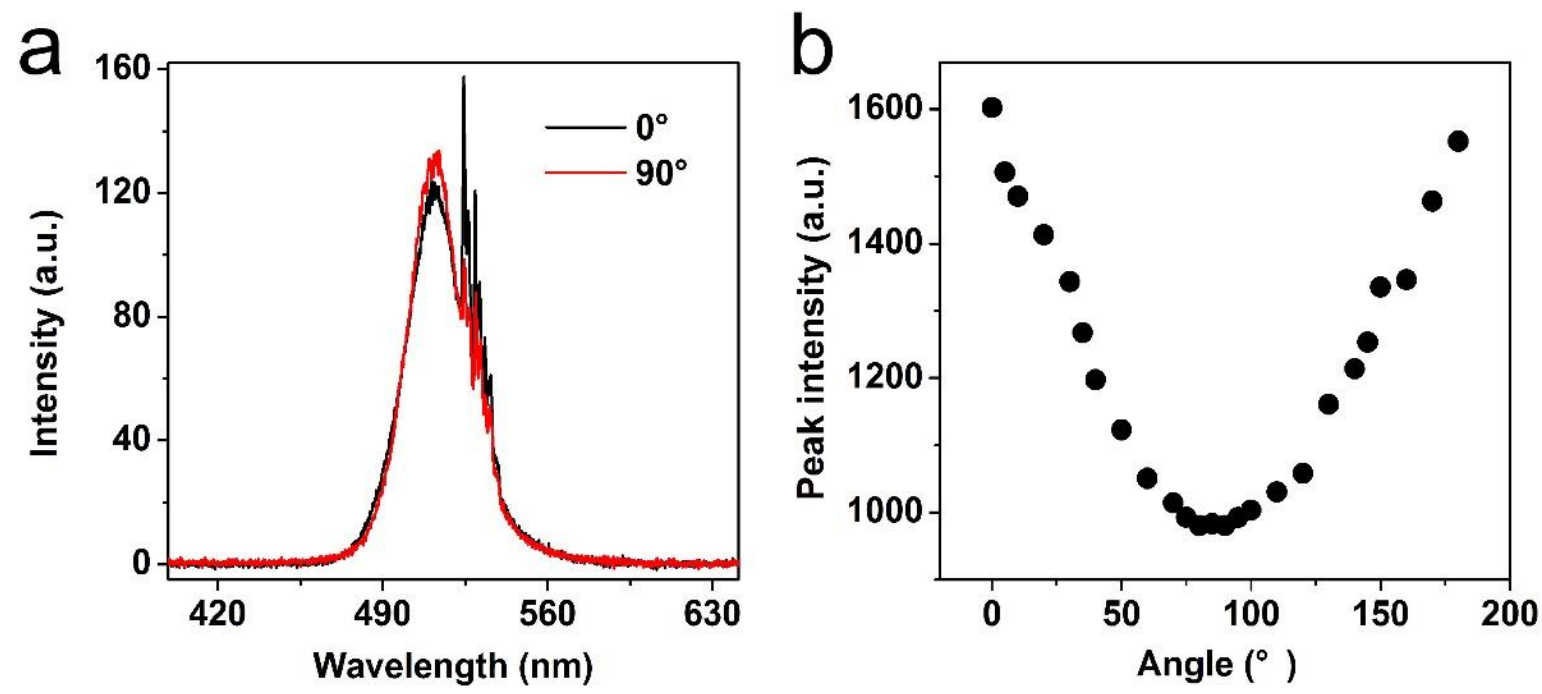

Figure S10. (a) The emission spectra of the device at $0^{\circ}$ and $90^{\circ}$. The strongest lasing emission is set at $0^{\circ}$ detection polarization. (b) The intensity of lasing peak at $524.4 \mathrm{~nm}$ with increasing degree of detection polarization. The pump power is fixed at $54 \mathrm{~W} / \mathrm{cm}^{2}$. 


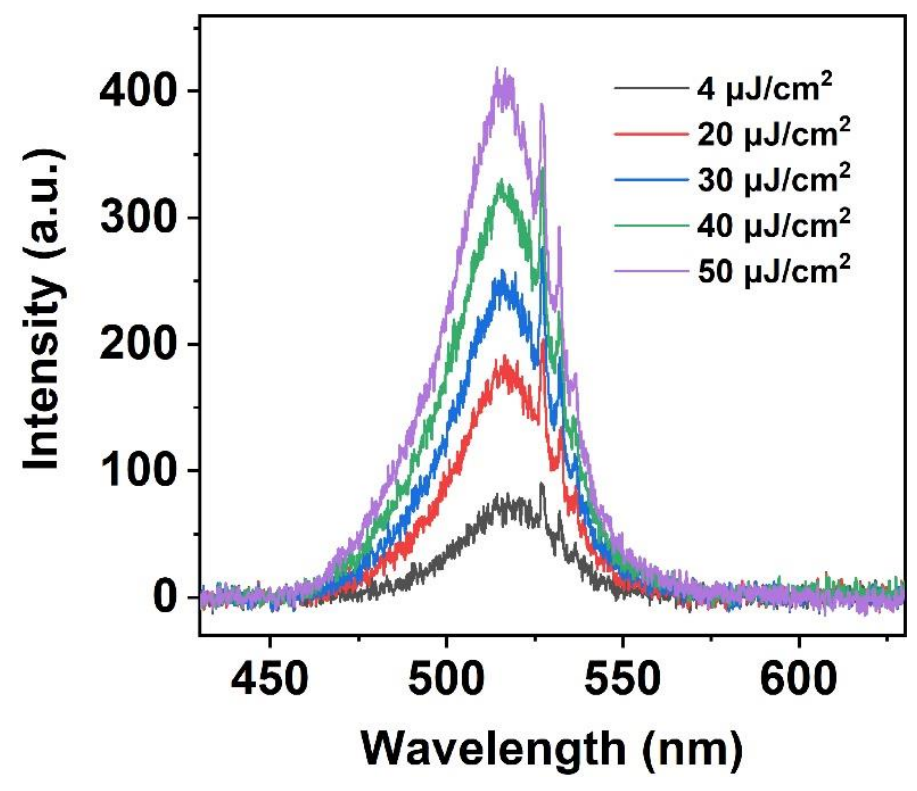

Figure S11. The lasing emission spectra of the green PQD-PAN film on the DFB cavity as a function of excitation energy. The pump source is femtosecond-pulsed laser.

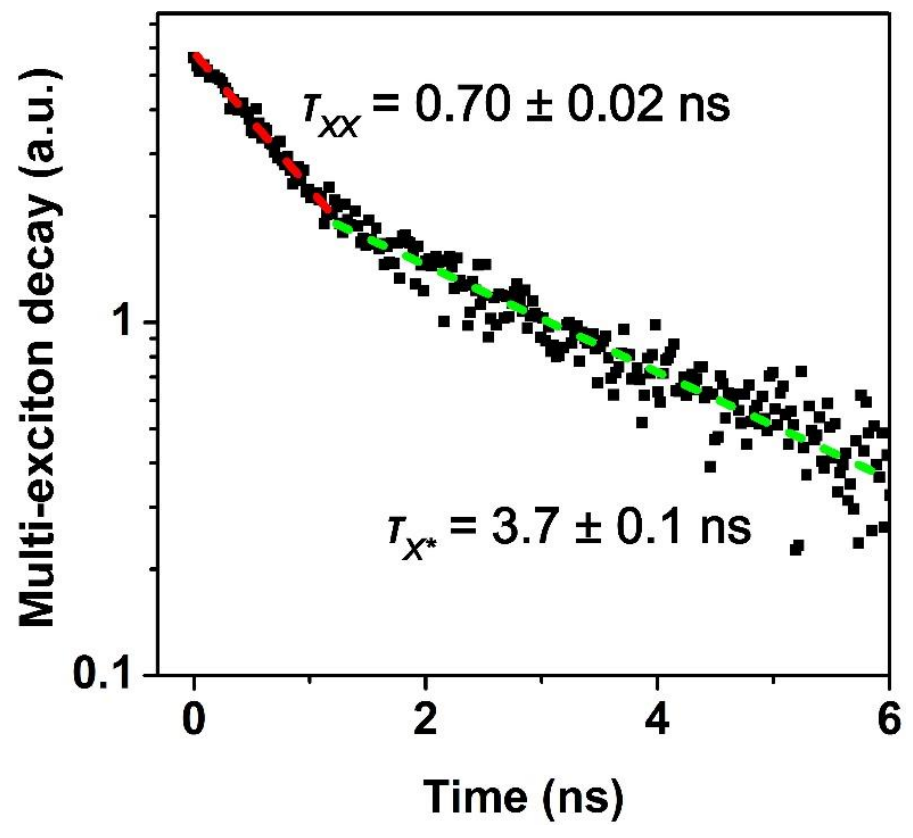

Figure S12. The dynamics components of biexciton or trion. Two clear decay times are observed. The fast and slow decay times are $0.70 \pm 0.02 \mathrm{~ns}$ and $3.7 \pm 0.1 \mathrm{~ns}$ respectively. The fast one attributes to the biexciton recombination, and the slow one corresponds to the trion recombination. 

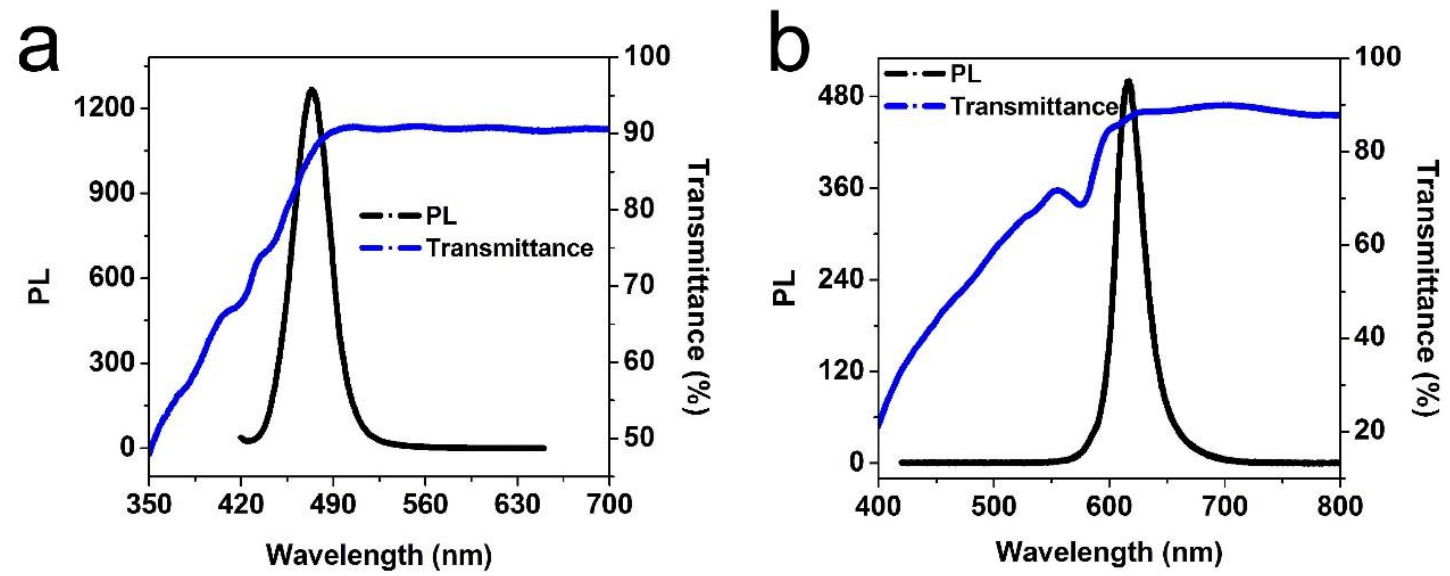

Figure S13. Transmittance and PL spectra of (a) blue and (b) red emissive PQD-embedded PAN films. The transparency of the blue and red film exceeds $90 \%$ and $87 \%$ respectively.

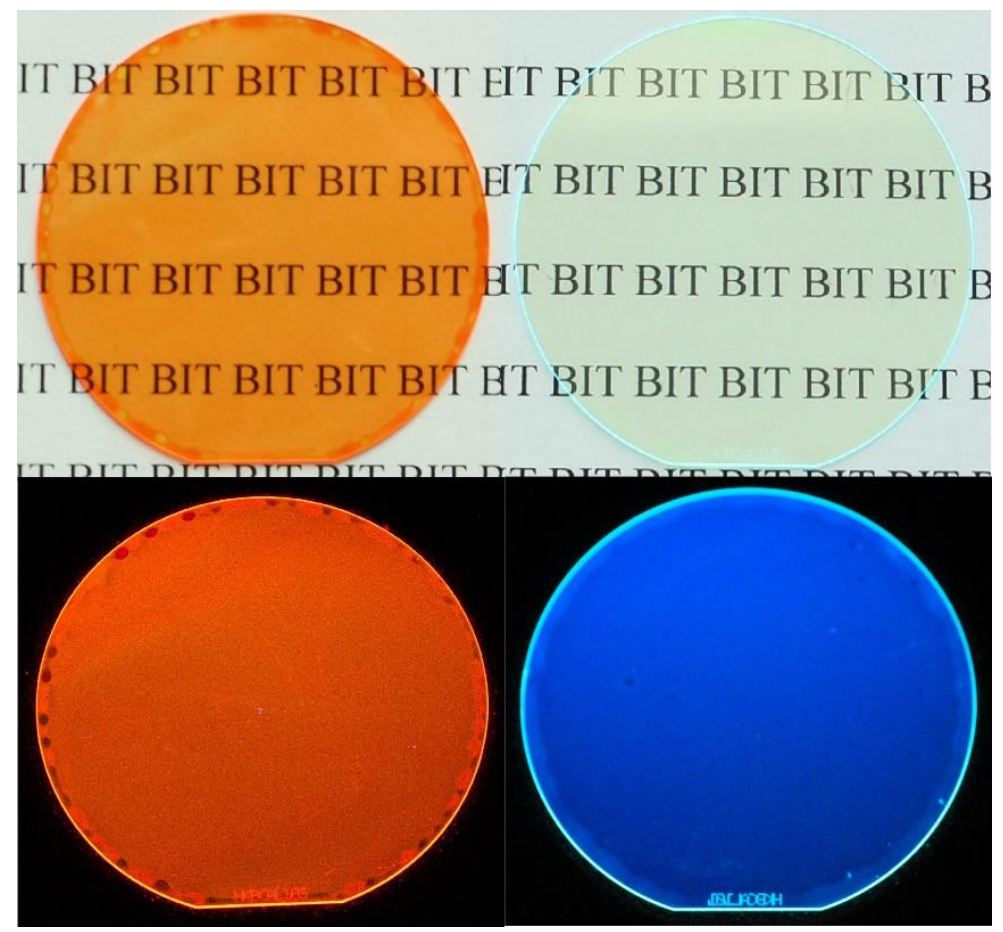

Figure S14. The optical image of the blue and red emissive PQD-embedded PAN films under daylight and UV light. 

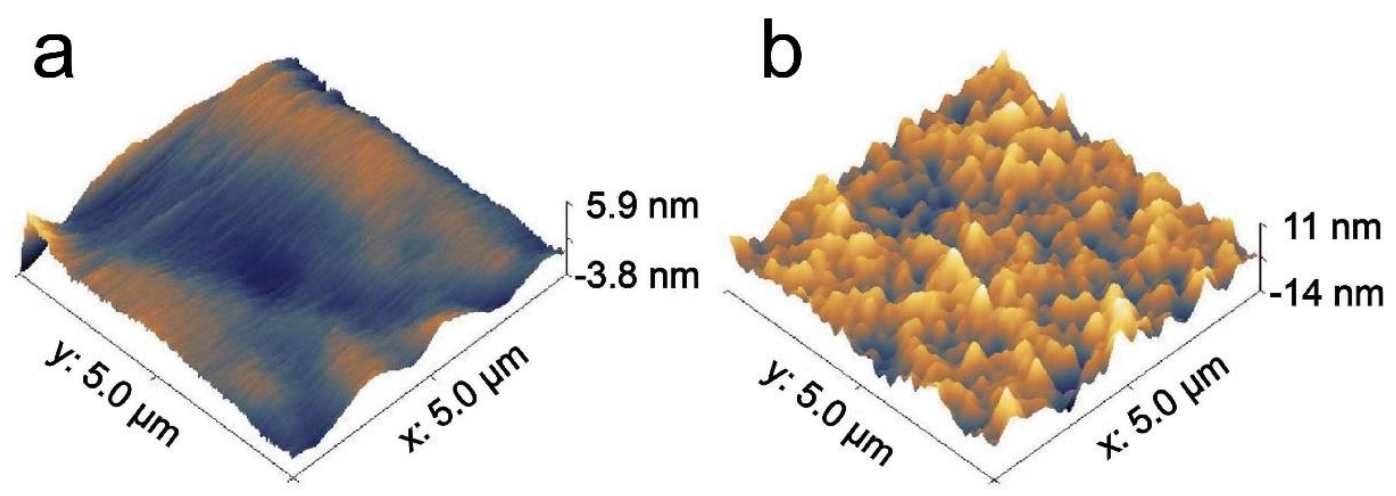

Figure S15. $5 \mu \mathrm{m} \times 5 \mu \mathrm{m}$ AFM images of the $\sim 2.5 \mu \mathrm{m}$ thick (a) blue and (b) red emissive PQDembedded PAN films spin-coated on quartz glass. The root-mean-square roughness is $0.9382 \pm 0.2786$ and $3.004 \pm 0.542 \mathrm{~nm}$, respectively.

\section{REFERENCES}

(1) Zhou, Q.; Bai, Z.; Lu, W. G.; Wang, Y.; Zou, B.; Zhong, H. In Situ Fabrication of Halide Perovskite Nanocrystal-Embedded Polymer Composite Films with Enhanced Photoluminescence for Display Backlights. Adv. Mater. 2016, 28, 9163-9168.

(2) Jia, W.; Cao, Z.; Wang, L.; Fu, J.; Chi, X.; Gao, W.; Wang, L. W. The Analysis of A Plane Wave Pseudopotential Density Functional Theory Code on A GPU Machine. Computer Phys. Commun. 2013, 184, 9-18.

(3) Jia, W.; Fu, J.; Cao, Z.; Wang, L.; Chi, X.; Gao, W.; Wang, L. W. Fast Plane Wave Density Functional Theory Molecular Dynamics Calculations on Multi-GPU Machines. J. Comput. Phys. 2013, 251, 102-115. 
(4) Han, Z.; Fina, A. Thermal Conductivity of Carbon Nanotubes and Their Polymer Nanocomposites: A Review. Prog. Polym. Sci. 2011, 36, 914-944.

(5) Chen, H.; Ginzburg, V. V.; Yang, J.; Yang, Y.; Liu, W.; Huang, Y.; Du, L.; Chen, B. Thermal Conductivity of Polymer-Based Composites: Fundamentals and Applications. Prog. Polym. Sci. 2016, 59, 41-85.

(6) Grim, J. Q.; Christodoulou, S.; Di Stasio, F.; Krahne, R.; Cingolani, R.; Manna, L.; Moreels, I. Continuous-Wave Biexciton Lasing at Room Temperature Using Solution-Processed Quantum Wells. Nat. Nanotech. 2014, 9, 891-895.

(7) Jia, Y.; Kerner, R. A.; Grede, A. J.; Rand, B. P.; Giebink, N. C. Continuous-Wave Lasing in an Organic-Inorganic Lead Halide Perovskite Semiconductor. Nat. Photonics. 2017, 11, 784788.

(8) Chien H. C.; Cheng C. Y.; Mao M. H. Continuous Wave Operation of $\mathrm{SiO}_{2}$ Sandwiched Colloidal CdSe/ZnS Quantum-Dot Microdisk Lasers. IEEE J. Sel. Top. Quant. 2017, 23, 1500405.

(9) Evans, T. J. S.; Schlaus, A.; Fu, Y.; Zhong, X.; Atallah, T. L.; Spencer, M. S.; Brus, L. E.; Jin, S.; Zhu, X. Y. Continuous-Wave Lasing in Cesium Lead Bromide Perovskite Nanowires. Adv. Optical Mater. 2018, 6, 1700982.

(10) Fan, F.; Voznyy, O.; Sabatini, R. P.; Bicanic, K. T.; Adachi, M. M.; McBride, J. R.; Reid, K. R.; Park, Y. S.; Li, X.; Jain, A.; Quintero-Bermudez, R.; Saravanapavanantham, M.; Liu, M.; Korkusinski, M.; Hawrylak, P.; Klimov, V. I.; Rosenthal, S. J.; Hoogland, S.; Sargent, E. H. Continuous-Wave Lasing in Colloidal Quantum Dot Solids Enabled by Facet-Selective Epitaxy. Nature 2017, 544, 75-79.

(11) Yang, Z.; Pelton, M.; Fedin, I.; Talapin, D. V.; Waks, E. A Room Temperature Continuous- 
Wave Nanolaser Using Colloidal Quantum Wells. Nat. Commun. 2017, 8, 143.

(12) Wu, K.; Park, Y. S.; Lim, J.; Klimov, V. I. Towards Zero-Threshold Optical Gain Using Charged Semiconductor Quantum Dots. Nat. Nanotech. 2017, 12, 1140-1147.

(13) Li, Z.; Moon, J.; Gharajeh, A.; Haroldson, R.; Hawkins, R.; Hu, W.; Zakhidov, A. A.; Gu, Q. Room-Temperature Continuous-Wave Operation of Organometal Halide Perovskite Lasers. ACS Nano 2018, 12, 10968-10976. 